This item was submitted to Loughborough's Research Repository by the author.

Items in Figshare are protected by copyright, with all rights reserved, unless otherwise indicated.

\title{
Arthroscopic repair of isolated type II superior labrum anterior-posterior lesion
}

PLEASE CITE THE PUBLISHED VERSION

http://dx.doi.org/10.1007/s00167-008-0629-4

\section{PUBLISHER}

(C) Springer

VERSION

AM (Accepted Manuscript)

\section{PUBLISHER STATEMENT}

This work is made available according to the conditions of the Creative Commons Attribution-NonCommercialNoDerivatives 4.0 International (CC BY-NC-ND 4.0) licence. Full details of this licence are available at: https://creativecommons.org/licenses/by-nc-nd/4.0/

\section{LICENCE}

CC BY-NC-ND 4.0

\section{REPOSITORY RECORD}

Yung, Patrick Shu-Hang, Daniel Tik-Pui Fong, Ming-Fat Kong, Chun-Kwong Lo, Kwai-Yau Fung, Eric P.Y. Ho, Derwin King-Chung Chan, and Kai-Ming Chan. 2019. "Arthroscopic Repair of Isolated Type II Superior Labrum Anterior-posterior Lesion”. figshare. https://hdl.handle.net/2134/21247. 


\section{Springer}

Draft manuscript for review

Arthroscopic repair of isolated type II superior labrum anterior posterior lesion

\begin{tabular}{|r|l|}
\hline \multicolumn{1}{|c|}{ Journal: } & Knee Surgery, Sports Traumatology, Arthroscopy \\
\hline Manuscript ID: & KSSTA-08-0244.R3 \\
\hline Manuscript Type: & Original Article \\
\hline Date Submitted by the Author: & n/a \\
\hline Complete List of Authors: & $\begin{array}{l}\text { Yung, Patrick; Alice Ho Miu Ling Nethersole Hospital, Orthopaedics and Traumatology } \\
\text { Fong, Daniel; The Chinese University of Hong Kong, Orthopaedics and Traumatology } \\
\text { Kong, Ming-Fat; Alice Ho Miu Ling Nethersole Hospital, Orthopaedics and Traumatology } \\
\text { Lo, Chun-Kong; Alice Ho Miu Ling Nethersole Hospital, Orthopaedics and Traumatology } \\
\text { Fung, Kwai-Yau; Alice Ho Miu Ling Nethersole Hospital, Orthopaedics and Traumatology } \\
\text { Chan, Kai-Ming; The Chinese University of Hong Kong, Orthopaedics and Traumatology }\end{array}$ \\
\hline Keywords: & \begin{tabular}{l} 
Shoulder, SLAP, sports medicine, arthroscopy, surgery \\
\hline
\end{tabular} \\
\hline
\end{tabular}

\section{S) scholarONE \\ Manuscript Central}


$\underline{\text { Title Page }}$

Title: Arthroscopic repair of isolated type II superior labrum anterior posterior lesion

Authors: $\quad$ Patrick Shu-Hang YUNG ${ }^{1,2,3}$, Daniel Tik-Pui FONG ${ }^{2,3}$, Ming-Fat KONG ${ }^{1}$, Chun-Kong LO ${ }^{1}$, Kwai-Yau FUNG ${ }^{1,2}$, Kai-Ming $\mathrm{CHAN}^{2,3}$

Institutions: $\quad{ }^{1}$ Department of Orthopaedics and Traumatology, Alice Ho Miu Ling Nethersole Hospital, Tai Po, Hong Kong, China.

${ }^{2}$ Department of Orthopaedics and Traumatology, Prince of Wales Hospital, Faculty of Medicine, The Chinese University of Hong Kong, Hong Kong, China.

${ }^{3}$ The Hong Kong Jockey Club Sports Medicine and Health Sciences Centre, Faculty of Medicine, The Chinese University of Hong Kong, Hong Kong, China.

Running title: Arthroscopic repair of isolated Type II SLAP lesion

Name and address or corresponding author

Name: $\quad$ Kai-Ming CHAN

Address: Department of Orthopaedics and Traumatology, Faculty of Medicine, The Chinese University of Hong Kong, Hong Kong, China

Telephone: (852) 26322728

Facsimile: (852) 26463020

E-Mail: $\quad$ kaimingchan@cuhk.edu.hk 


\begin{abstract}
The effectiveness of arthroscopic repair of type II superior labrum anterior posterior lesion (SLAP) was unclear as previous studies examined this treatment with patients of combined types of SLAP lesions. To address this research gap, we evaluated the clinical and functional outcomes of arthroscopic repair for 16 patients $($ Mean $=24.2$, S.D. $=6.5)$ with clinical evidence of isolated type II SLAP lesion. After having arthroscopic stabilizations with Bioknotless suture anchors (Mitek), the patients were offered post-operative rehabilitation programs (e.g., physiotherapy) for 6 months. The symptoms of SLAP lesion and the functions of the shoulder were assessed preoperatively and 28-month post-operatively by O'Brien test, Speed test, Yergason test, and University of California at Los Angeles rating for pain and function of the shoulder. Wilcoxon Signed Ranks test and McNemar test were employed to analyze the difference between assessment in pre-operation and post-operation phases. The result showed that patients' shoulder functions improved (UCLA Shoulder Score), and symptoms of SLAP lesion reduced (O'Brien test, Speed test, and Yergason test) significantly $(p<0.05)$. Time for returning to play with pre-injury level was in average 9.4 months (range: 4-24), and no complication or recurrence was detected. We concluded that arthroscopic repair is an effective operation of type II SLAP lesion with good clinical and functional outcomes, however, athletes with high demand of overhead throwing activities are likely to take longer duration of rehabilitation attain fully recovery.
\end{abstract}

Keywords: Shoulder, SLAP, sports medicine, arthroscopy, surgery 


\section{Introduction}

Historically, "dead arm" has been regarded as a career ending affliction for overhead throwing athletes, as the causes and treatment of this pathology were almost unknown until the recent advent of arthroscopy [3,5]. Advances in imaging techniques and the rapid developments and evolutions of arthroscopic surgery enabled surgeons to understand a lot of previously unknown shoulder pathologies and to treat them accordingly during the past two decades. The story of the "dead arm" was begun to be uncovered 23 years ago, when Andrews et al [2] carried out initial investigation and suggested that injuries in superior labrum-bicep anchors in glenoidhumeral joints was the major cause of the pathology. By then, there has been an increasing number of orthopedic and radiology literatures focusing on the diagnosis and treatment of this shoulder pathology.

Synder et al [24] defined this type of shoulder pathology, named superior labrum anterior posterior (SLAP) lesion, as the superior labrum-biceps tendon complex disruptions which involved of tearing and separation of the superior labrum, or both; in addition, the tearing or separation is developed posteriorly from the biceps tendon insertion and extending anteriorly. Besides, Snyder et al [24] pointed out that this lesion is not uncommon, while $6 \%$ of his arthroscopic operations were related to SLAP lesion. Maffet et al [14] also revealed that SLAP lesion presented in $12 \%$ of his shoulder arthroscopy cases.

As we have previously mentioned, one of the interesting phenomena of the prevalence of SLAP lesion is that, the pathology is usually specified to athletes of particular sports, who developed the lesions from a compression force of the injured shoulder with the arm flexed and abducted upon a fall, or upon a traction injury to the arm [14]. Whereas Warner et al [28] described another group of patients of SLAP lesion developed the lesions by repetitive overhead pitching activities. This is probably resulted from eccentric loading of the bicep with a sudden increase in the tensile force of the anchor, during the cocking phase of a throw, and lesion is developed either by repetitive micro trauma or single major trauma.

From all his arthroscopic cases, Synder et al [24] identified four major types of injuries (outstretching injury, traction, direct trauma, external rotation) causing this superior glenoid labrum lesion, and thus categorize SLAP lesions into four types (Type I, II, III, IV) in terms of the mechanism of the injuries and arthroscopic evaluations. In the past decade, while more and more studies investigated the pathologies and treatment, ten types of SLAP lesions have been identified $[3,12,16,19,20]$. Among the ten types of SLAP lesion, type II lesion is the most common one, which is defined by "superior labral fraying with stripping of the superior part of the labrum and attached biceps tendon from the underlying glenoid cartilage" [3,19]. Later on, Morgan et al [16] further identified three distinct sub types of type II SLAP lesions (Type II A, B, and C). In particular, type IIA, IIB, and IIC represent anterosuperior labral lesion, posterosuperior lesion, and superior lesion with both anterior and posterior components respectively.

Reported treatment for SLAP lesion have found to be mixed, which ranged from 
debridement of the lesions (for type I and III) [1,6] to surgical repair (for type II and IV lesion) $[8,11,22,25]$. Moreover, the surgical treatments of these various types of SLAP lesion differ from each other in terms of the fixation methods and operative devices, such as staples, metallic and absorbable suture anchors or tacks. As a result, it is difficult to justify the overall surgical outcomes of SLAP lesion while inconsistencies about the type of injuries and surgical treatment present within a single study. Although there have been dozens of relevant clinical studies about surgical treatment of SLAP lesion over the decades, most previous studies only consisted of SLAP cases with the combinations of SLAP lesions and other related intra-articular pathologies, like Bankart lesion or rotator cuff tear. Thus, the results of these studies may be confounded by subject effects, and the effectiveness of the surgical treatments of type II SLAP lesion was unclear.

To address this research gap, the purpose of this study is to evaluate the clinical and functional outcome of our patients, with isolated Type II SLAP lesion of the shoulder, who underwent arthroscopic stabilization as the surgical treatment of SLAP lesion, from July 2003 to March 2005. Thus, the clinical and functional outcomes of arthroscopic treatment to isolated Type II SLAP lesion can be examined.

\section{Methods}

\section{Participants}

In order to obtain our target sample, we revealed all patients of the Prince of Wales Hospital who showed clinical evidence of isolated Type II SLAP lesion during the period (July 2003-October 2005). They were also required to fulfill the following inclusion criteria:

1. No history of shoulder dislocation or subluxation.

2. No intra-articular injuries of Type II SLAP lesion

3. No neurological complications affecting mobility and physical sensation

4. No previous surgery over the same shoulder

5. Non work-compensation cases

6. Age less than 40

After employing a prospective cohort study using magnetic resonance imaging (MRI) arthrogram, 23 cases of Type II SLAP lesion satisfied the above criteria, and were advised by their physicians to undergo arthroscopic stability operations. Among the cases, 20 patients agreed to participate after being consented about the study purposes and their rights of participating the study. However, 4 patients were found to have concomitant intra-articular pathologies during arthroscopic examination, so they were then excluded from our study. Finally, our sample consists of 13 male and 3 female participants whose ages ranged from 15 to $38($ Mean $=24.2$, S.D. $=6.5)$. 
Among the 16 participants, fifteen had type II lesion on their right shoulders, and only 1

80 had the lesion on the left shoulder. Before injury, 13 participants were actively involved in overhead sports (e.g., tennis, handball, badminton) in either elite or recreational levels, two others were believed to get injured by lifting heavy weights, and one was injured by landing with an outstretched hand after a fall. All patients received operation within 1 month after their first visit to the clinic, and were given physiotherapy and analgesics treatment after their operations.

\section{Operative procedures}

After receiving general anaesthesia, patients were put into lateral decubitus position under traction of three to four kilograms. Diagnostic arthroscopy was performed via the standard posterior portal. Superior labrum was carefully tested with an arthroscopic probe through an anterior-superior portal. Not uncommonly a Type II SLAP lesion looks normal upon arthroscopic examinations, but became obvious when it is tested with an arthroscopic probe for its stability. Other associated intra-articular structures, including the rest of the labrum, bicep tendon, the anterior, inferior and posterior capsules, undersurface of the rotator cuff, and the articular cartilage, were throughout examined.

After identification of the SLAP lesion, an arthroscopic shaver or a burr was introduced via the antero-superior portal to debride the superior glenoid bone edge till the good bleeding raw area was prepared. Based on particular extent of the SLAP tear, we used different portals to fix the lesion for the patients. For anterior extension of the SLAP tear, we tackled lesions via the anterior-superior portals. For posterior extension of the lesion, we used trans-rotator cuff portals, as suggested by O'Brien et al [10]. These portals were approximately $1-2 \mathrm{~cm}$ lateral to the lateral edge of the acromion. Spectrum suture passer device (Linvatec) was used to deliver a PDS-1 suture via either superior labrum or working portals, which depended upon the location of the lesion. The free distal limb of the PDS-1 suture was retrieved through the anterior-superior portal, or an anterior-mid glenoid portal.

Except two cases, all patients' Type II SLAP tears were fixed with bioabsorbable knotless suture anchors (Bioknotless Anchor, Mitek) by the technique described by Thal [17], at 2, 1, 11 or 10 o'clock region according to the pattern of labral tear. The utility loop suture ends of the Bioknotless anchors were then loaded into the proximal open loop end of the PDS-1, and were pulled from the other portal through the labral tissue using "poor man shuttle" technique. Tension was maintained along both suture ends while passing the anchor in. After the utility loop passed via the superior labrum, one of the strands of the loop was captured under the anchor prongs, and then the anchor was driven into the drill hole under direct vision, until the anchor was completely engaged. Precautions were taken to ensure that the suture loop was not twisted while the anchor was being inserted, and the anchor was not inserted too deeply into the drill hole, as these two situations may lead to the abrasion of the sutures, and thus breakage. The number of anchors used in this study ranged from 2 to 4 $($ Mean $=2.6$, S.D. $=.7)$, while two to three absorbable anchors are usually enough to provide 
118 a stable repair to most SLAP lesions. The operation time, type of SLAP lesions, number of 119 anchors and hospitalized days are listed in table 1.

120 Post-operative rehabilitation

121 Post-operatively, the patients followed a standard rehabilitation protocol of shoulder 122 lesion surgery [9]:

123

124

125

126

127

128

129

130

131

132

133

134

135

136

137

138

139

140

141

142

143

144

145

146

147

148

149

150

151

152

153

154

155

156

Week 1: - Sling immobilization at all times

Week 2-3: - Codman circumduction

- Passive range of motion (0-90 abduction)

- External rotation adduction

- No external rotation in abduction

Week 4-6: - Discontinue sling

- Progressive passive range of motion (PROM) to full in all planes

- Passive posterior capsular and internal rotation stretching

- Passive and manual scapulothoracic mobility program

- External rotation in abduction

Week 7-16 - Progressive strengthening of the rotator cuff, scapular stabilizers and biceps

Month 4-6: - Internal throwing program on level surface

- Return to full throwing activities

In addition, during the first 4 post operative weeks, the patients were advised to restrain from external rotation in the abduction for reducing the possibilities of re-injury due to peel-back mechanism. Finally, patients were also advised not to resume throwing sports activities until improvement in their shoulders' range of motion, rotator cuff strength, and biceps strength, were observed compared to their preoperative status. All the patients were able to comply with the rehabilitation program and attend follow-up reassessment regularly in our clinic, with an average follow-up of 27.6 \pm 2.6 months (range: $24-31$ ). Assessments and analysis

Participants were asked to undergo O'Brien test [17] for detecting SLAP lesion. O'Brien test [17] is a newly developed compression test to diagnose labral tears and pathological conditions of the acromioclavicular joint, and was clinically found to be highly reliable in detecting SLAP injuries. In the test, patients are asked to sit in an upright position and elevate their shoulder with the arm in full extension. Examiner then adducts the arm for $10^{\circ}$, and rotates internally until the thumb points downwards. The arm in the position is then pushed downwards repeatedly with supination of the arm. A sensation of pain or clicking inside the joint indicates the presence of labral or SLAP tear.

Besides, Speed test [9] and Yergason test [30] were performed for assessing patients' biceps tendon instability or tendonitis. These two tests are not specific for detecting SLAP lesion, as recent studies showed that Speed test and Yergason test are not sensitive clinical 
tests for detecting SLAP lesion [10] in particular shoulder region (e.g., posterior SLAP

158

159

160

161

162

163

164

165

166

167

168

169

170

171

172

173

174

175

176

177

178

179

180

181

182

183

184

185

186

187

188

189

190

191

192

193

194

195

lesion for Speed test). However, we decide to include these two tests to provide additional information as they are classical test for diagnosing shoulder injuries, and it is not clear about their sensitivities in detecting lesion specifically for type II lesion.

Moreover, The University of California at Los Angeles rating for pain and function of the shoulder [7] was adopted to access the patients' shoulder joins functions. The scale consists of five items. The two items corresponding for the range and strength (manual muscle-testing) of active forward flexion are graded by physicians in a 6-point-likert scale ( 0 indicates worst condition, and 5 indicates best condition). The other three corresponding for the pain and function of shoulders, and satisfaction towards their shoulders are self-reported by patients. Pain and function are measured in 10-point-likert scale with the scores of 1 and 10 representing the worst and best condition respectively. The satisfaction score is accessed in a 6-point-likert scale as pain and function do. Therefore, the scores of the five items composite into a UCLA Shoulder Score [6] with a maximum of 35 marks, while patients receiving 34-35 marks, 29-33 marks, and 29 marks or lower are classified as having excellent, good, and poor shoulder function respectively [6].

These assessments were performed preoperatively (the day before surgery) and postoperatively $(27.6 \pm 2.6$ months after the surgery) by two orthopaedics specialists with more than 15 years of clinical experience. In addition, at the final postoperative assessment, participant were asked to report their pre-injury activity levels, injury mechanisms, duration of symptoms, and the time required to return to pre-injury activity levels. (See table 2)

In this study, the effect of the operative treatment of type II SLAP lesion were examined by comparing the difference of test scores patients obtained in their pre-operative assessment and post-operative assessment, in which participant served as their own control. McNemar test (for categorical variables) was employed to examine the categorical variable of O'Brien test. As the problem of non-normality, which violates the assumptions of t-test, might possibly presented in our small sample, we examined the normality of continuous variables like UCLA shoulder scores and sub-scores by Shapiro-Wilk tests to determine whether Wilcoxon Signed Ranks test (for skewed population) or paired sample t-test (for normally distributed sample) should be used for analyzing the continuous variables. Significant difference of the test scores between pre-operative assessment and post-operative assessment were indicated by significant $p$ values $<.05$.

\section{Results}

\section{Intra-operative findings}

The operations took 71 minutes on average (S.D. = 14; range: 52-105). Arthroscopic screening based on the morphology of the Type II SLAP lesions revealed that, there were seven cases of superior-anterior lesion (Type IIA), five cases of superior-posterior lesion (Type IIB), and four cases of combined superior-anterior-posterior lesions (Type IIC), where 
196 the lesions were located around the bicep anchor region, namely the 12 o'clock position.

197 The lesions were repaired with two to four anchors (Mean=2.6, SD =0.7), distributed

198 equally from two to ten o'clock area. Intra-operatively, there were two episodes of breakage

199 of anchors during the insertion due to incorrect approaching angle with respect to the drill

200 hole. There were also two episodes of suture abrasion and then breakage noted

201 intra-operatively. One of the cases was because of twisting of the suture loop during anchor

202 insertion, and the other case was related to too deep suture anchorage into the glenoid bone.

203 Both problems were noted intra-operatively and another suture anchor was placed right next

204 to it for repairing the labrum.

205 Outcome assessment

206 McNemar test showed that significant difference was observed between the test-retest 207 assessment of O'Brien test, Speed test, and Yergason test $(p<.05)$. Patients with a positive 208 signs of O'Brien test Speed test, and Yergason test significantly reduced in the 209 post-operative phase.

210 Shapiro-Wilk tests revealed that all UCLA shoulders scores in the pre-operative 211 assessment, and post-operative assessment were significantly skewed $(\mathrm{p}<.05$, despite the 212 post-operative UCLA total score ( $p=.06$ ). Therefore, Wilcoxon Signed Ranks test was used, 213 and the result showed that significant difference were observed in all dimensions of UCLA 214 Shoulder assessment scores, and thus, the composite score of UCLA $(p<.05)$ between the 215 pre-operative and post operative assessment. Specifically, the shoulders' pain, function, 216 active forward flexion, strength, satisfaction, and overall function (composite scores) of 217 their shoulders significantly increased in the post-operative phase $(p<.05)$. Details are 218 shown in table 2.

219 In addition, based on the grading system of UCLA assessment, 100\% of patients in the 220 pre-operative phase were categorized as having poor shoulder function (Mean=18.1, $221 \mathrm{SD}=3.3$ ), while in the post-operative assessment, $31.3 \%, 43.8 \%$, and $25.0 \%$ of patients were 222 categorized as having excellent (Mean=35.0, $\mathrm{SD}=0$ ), good (31.6, $\mathrm{SD}=1.9$ ), and poor 223 function $(26.3, \mathrm{SD}=2.1)$. See table 2 for details.

224

225 Discussion

226 The purpose of the study was to examine the effects of surgical treatment of isolated 227 type II lesion. The results showed that arthroscopic repair of isolated Type II SLAP lesion 228 achieved excellent to good results in majority of patients $(\mathrm{N}=14,87.5 \%)$, which was 229 comparable to other series, with a mixed combinations of pathologies associated to SLAP 230 lesion, as reported by different authors [8,11,14,22,24,25,28] (80-100\% good outcomes).

\section{Clinical outcomes}

232 Compared to the results of the pre-operative assessments, positive signs from O'Brien 233 test, Speed test, and Yergason test significantly reduced in the post-operative assessment, 234 providing supporting evidence for the effectiveness of the arthroscopic treatment of type II 
235 SLAP lesion. UCLA scores increased sharply from an average of $18.1(\mathrm{SD}=3.3)$ to 31.3 236 (SD = 3.7), which indicated the shoulder function improved significantly after the surgical 237 treatment. According to the categorization of UCLA shoulder assessment [7], the percentage 238 of patients having poor shoulder function reduced from $100 \%$ at the pre-operative assessment, to only $25 \%$ at the post-operative assessment. While the UCLA total scores of these $25 \%$ patient on average increased to $26.3(\mathrm{SD}=2.1)$, a value closed to good function category. These evident support the effectiveness of arthroscopic treatment of type II SLAP lesion.

Despite of clinical assessments, the recovery duration patients need for returning their pre-injury activities levels is also an important success indicator towards treatment. In our study, all patients started to regain active and passive range of motions from the fourth weeks after the operations. At six months after the operation, 14 patients $(88 \%)$ regained active range of motion within 9 months post-operatively. All patients regained their full range of motion upon the final assessment without complications. Filed and Savoie [8] reported that all their patients returned to athletic activities without limitations. However, we argue that the recovery of SLAP lesion after the operation might depend on pre-injury activity levels of the patients. In our study, elite athletes who required frequent overhead activity required longer duration of rehabilitation and time to return to pre-injury activity level. Eleven patients $(69 \%)$ regained pre-injury activity level within nine months after the operation (average $=7.0$ months), and four patients, who previously performed intense and frequent overhead activities ( 2 tennis, 1 badminton, and 1 cricket) in elite levels returned to their pre-injury levels after an obviously longer period of post-operation rehabilitation (Mean = 11.0 months). The remaining one elite handball athlete could not resume his pre-injury activity level at 24 months after the operation, because of residual pain and weakness of the supraspinatus. In line with our study, Burkhart et al [4] and Kim et al [11] both reported that throwing athletes produced statistically inferior results for SLAP lesion than non-throwing athletes. They usually took longer period of time to recover, and sometimes even failed to show return to their pre-injury performance.

Though there was significant improvement in terms of clinical test scores in the post-operative phase, it is important to note that reported pain persisted in minority of cases $(\mathrm{N}=3)$, including night pain. MRI were employed post-operatively for these patients and revealed that their SLAP lesions did not present, which evidenced by the marked difference in supraspinatus tendinosis, and articular side tear of the supraspinatus tendon. We suggested that the pain could be explained by the occult pathology beyond our clinical detection, and thus residual pain and signs of impingement syndrome persisted even though we had tackled the SLAP lesion. Further studies may examine the operative pain phenomenon, in terms of the causes, screening methods, and treatments.

\section{Surgical treatments of SLAP lesion}


274 the surgical treatment of SLAP lesion (e.g., metal screws, metal staples, suture, and 275 absorbable tack or screw), however, dozens of argument and concerns have been pointed out 276 regarding the use of these metal implants. For examples, it has been suggested that metal 277 implant may possibly injure the articular cartilage upon insertion, and subsequently protrude 278 into the joint, or become loosen [19,31,32]. For this reason, surgeons began to put more 279 attention in using absorbable sutures. Field and Savio [8], who used absorbable sutures, 280 reported good clinical results in the operation of SLAP lesion. Similarly, Snyder el al [23] 281 and Burkhart et al [4] demonstrated encouraging results by using absorbable tacks, but both 282 of them illustrated problems of tack fragment which required removal post-operatively. 283 There have also been concerns over the development of synovitis [4], and possibly bony 284 lysis [23] at the anchor site. In this study, we used Bioknotless suture anchors (Mitek) as 285 described by Thal [27]. It has been reported to provide a secure process, low profile of repair, 286 and improved healing potentials [27], as part of the tissue is pulled into the drill hole rather 287 than on the top of the anchor. Moreover, it avoids the added complexities of arthroscopic 288 knot tying, thus, the processes of surgery become more efficient [27].

\section{Limitation}

290 In this study, O'Brien test was the only test which is specifically for detecting SLAP 291 lesion. Further studies should include more shoulder SLAP lesion test, such as 292 compression-rotation test and crank test, which have been suggested to be as sensitive as 293 O'Brien test in detecting SLAP lesion in general [21,22].

294 Although type II SLAP lesion is the most common type of SLAP lesion [15,19], 295 recruiting participants with isolated type two SLAP lesion was somehow difficult, as most 296 type II SLAP lesion cases were combined with either type of SLAP injuries or rotator cutoff 297 pathologies $[2,6,8,11,12,14,25,28]$, so the sample size was relatively small, which was one 298 of the major limitation in our study.

299 Conclusion

300 Our study showed that arthroscopic stabilization of Type II SLAP lesion is effective 301 treatments evidenced by good clinical and functional outcome in majority of patients. 302 However, it was observed that athletes with high demand of overhead throwing activities 303 were likely to take longer duration of rehabilitation attain fully recovery. 


\section{References}

305

1. Altchek DW, Warren RF, Wickiewicz TL (1992) Arthroscopic labral debridement. A three-year follow-up study American Journal of Sports Medicine 20(6):702-706

2. Andrews JR, Carson WG, Jr., McLeod WD (1985) Glenoid labrum tears related to the long head of the biceps American Journal of Sports Medicine 13(5):337-341

3. Burkart A, Imhoff AB, Roscher E (2000) Foreign-body reaction to the bioabsorbable suretac device Arthroscopy 16(1):91-95

4. Burkhart SS, Morgan CD, Kibler WB (2000) Shoulder injuries in overhead athletes. The "dead arm" revisited Clinics in Sports Medicine 19(1):125-158

5. Burkhart SS, Morgan CD, Kibler WB (2000). The disable throwing shoulder: Spectrum of pathology part I: Patholoanatomy and biomechanics. Arthroscopy 19(4):404-420

6. Cordasco FA, Steinmann S, Flatow EL (1993) Arthroscopic treatment of glenoid labral tears American Journal of Sports Medicine 21(3):425-431

7. Ellman H, Hanker G, Bayer M (1986) Repair of the rotator cuff. End-result study of factors influencing reconstruction Journal of Bone and Joint Surgery - American Volume. 68(8):1136-1144

8. Field LD, Savoie FH, $3^{\text {rd }}$ (1993) Arthroscopic suture repair of superior labral detachment lesions of the shoulder American Journal of Sports Medicine 21(6):783-790; discussion 790

9. Gilecreest EL, Albi P (1939). Unusual lesions of muscles and tendons of the shoulder girdle and upper arm Surgery Gynecology \&Obstetrics 68:903-917

10. Holtby R, Razmjou H (2004) Accuracy of the speed's and Yergason's tests in detecting biceps pathology and SLAP lesions: comparison with arthroscopic findings Journal of Arthroscopic and Related Surgery 20(3): 231-236

11. Kim SH, Ha KI, Kim SH, et al. Results of arthroscopic treatment of superior labral lesions. Journal of Bone and Joint Surgery - American Volume. 2002;84-A(6):981-985.

12. Law BKY, Yung PSH, Ho PY, Chang JJ, \& Chan KM. The surgical outcome of immediate arthroscopic Bankart repair for first time anterior shoulder dislocation in young active patients . Knee Surgery, Sports Traumatology, Arthroscopy, 16: 188-193.

13. Liu SH, Henry MH, Nuccion SL (1996) A prospective evaluation of a new physical examination in predicting glenoid labral tears American Journal of Sports Medicine 24: $721-725$

14. Maffet MW, Gartsman GM, Moseley B(1995) Superior labrum-biceps tendon complex lesions of the shoulder American Journal of Sports Medicine 23(1):93-98

15. Mohana-Borges AVR, Chung CB, Resnick D (2003) Superior labral anteroposterior tear: classification and diagnosis on MRI and MR arthrography American Journal of 
343 Roentgenology 181:1449-1462

344 16. Morgan CD, Burkhart SS, Palmeri M, Gillespie M (1998) Type II SLAP lesions:

345 three subtypes and their relationships to superior instability and rotator cuff tears.

346 Arthroscopy 14:553-565

347 17. O'Brien SJ, Allen AA, Coleman SH (2002) The trans-rotator cuff approach to SLAP

348 lesions: technical aspects for repair and a clinical follow-up of 31 patients at a 349 minimum of 2 years. Arthroscopy 18(4):372-377.

350 18. O'Brien SJ, Pagnani MJ, Fealy S (1998). The active compression test: A new and 351 effective test for diagnosing labral tears and acromioclavicular joint abnormality 352 American Journal of Sports Medicine 26: 610-613

353 19. Resch H, Golser K, Theoni H. (1993) Arthroscopic repair of superior glenoid labral 354 detachment (the SLAP lesion) Journal of Shoulder and Elbow Surgery 2(3):147-155

355 20. Resnick D (1997) Shoulder In: Resnick D, Kang HS (eds.) Internal derangements of 356 357 358 359 360 361 362 363 364 365 366 367 368 369 370 371 372 373 374 375 376 377 378 379 joints: emphasis on MR imaging Philadelphia: Saunders $171-281$

21. Rhee YG, Lee DH, Lim CT (2005) Unstable isolated SLAP lesion: clinical presentation and outcome of arthroscopic fixation Arthroscopy 21(9):1099

22. Segmuller HE, Hayes MG, Saies AD (1997) Arthroscopic repair of glenolabral injuries with an absorbable fixation device Journal of Shoulder and Elbow Surgery 6(4):383-392

23. Snyder SJ, Banas MP, Karzel RP (1995) An analysis of 140 injuries to the superior glenoid labrum Journal of Shoulder and Elbow Surgery 4(4):243-248

24. Snyder SJ, Karzel RP, Del Pizzo W (1990) SLAP lesions of the shoulder. Arthroscopy 6(4):274-279

25. Speer KP, Warren RF, Pagnani M (1996) An arthroscopic technique for anterior stabilization of the shoulder with a bioabsorbable tack Journal of Bone and Joint Surgery - American Volume 78(12):1801-1807

26. Thal R (2001) Knotless suture anchor: arthroscopic bankart repair without tying knots Clinical Orthopaedics and Related Research 390:42-51.

27. Thal R (2001) A Knotless Suture Anchor: Technique for use in arthroscopic Bankart repair. Arthroscopy 17(2):213-218

28. Warner JJ, Kann S, Marks P (1994) Arthroscopic repair of combined Bankart and superior labral detachment anterior and posterior lesions: technique and preliminary results Arthroscopy 10(4):383-391

29. Wilkerson JP, Zvijac JE, Uribe JW (2003) Failure of polymerized lactic acid tacks in shoulder surgery Journal of Shoulder and Elbow Surgery 12(2):117-121

30. Yergason RM (1931) Supination sign. The Journal of Bone and Joint Surgery 13:160.

31. Yoneda M, Hirooka A, Saito S (1991) Arthroscopic stapling for detached superior glenoid labrum Journal of Bone and Joint Surgery - British Volume 73(5):746-750 
32. Zuckerman JD, Matsen FA, 3rd (1984) Complications about the glenohumeral joint 383 related to the use of screws and staples Journal of Bone and Joint Surgery 384 American Volume 66(2):175-180 
Table 1 - Demographics of the patients and details of operation and final assessment

\begin{tabular}{|c|c|}
\hline Age (years) & $24.2 \pm 6.5$ (range: $15-38)$ \\
\hline Gender (male/female) & $13 / 3$ \\
\hline Injured side (left/right) & $1 / 15$ \\
\hline Duration of symptoms at admission (weeks) & $18.3 \pm 6.1$ (range: $12-36)$ \\
\hline \multicolumn{2}{|l|}{ Pre-injury activity level } \\
\hline Overhead sports at national/varsity level & 5 \\
\hline Overhead sports at recreational level & 8 \\
\hline Non-overhead sports & 2 \\
\hline No sports & 1 \\
\hline \multicolumn{2}{|l|}{ Injury mechanism } \\
\hline Sports related with definite history of injury & 8 \\
\hline Sports related with repetitive overuse & 4 \\
\hline No-sports related & 2 \\
\hline Cannot be recalled & 2 \\
\hline \multicolumn{2}{|l|}{ Treatment sought } \\
\hline Physiotherapy & 16 \\
\hline Analgesics & 16 \\
\hline Operation time (minutes) & $71 \pm 14$ (range: $52-105)$ \\
\hline \multicolumn{2}{|l|}{ Type of SLAP lesions } \\
\hline IIA & 7 \\
\hline IIB & 5 \\
\hline IIC & 4 \\
\hline Number of anchors & $2.6 \pm 0.7$ (range: $2-4)$ \\
\hline Number of days of hospital stay (days) & $1.2 \pm 0.4$ (range: $1-2)$ \\
\hline Time for the final outcome assessment (months) & $27.6 \pm 2.6$ (range: $24-31)$ \\
\hline Time for return to play with pre-injury level (months) & $9.4 \pm 5.2($ range: $4-24)$ \\
\hline
\end{tabular}


Table 2 - Pre-operative assessment, final outcome and results of statistical tests.

\begin{tabular}{|c|c|c|c|}
\hline & Pre-operative & Final outcome & Statistical results \\
\hline \multicolumn{4}{|l|}{ Clinical test $(+/-)$} \\
\hline Speed test & $10 / 6$ & $4 / 12$ & $p<0.05^{1}$ \\
\hline Yergason test & $9 / 7$ & $2 / 14$ & $p<0.05^{1}$ \\
\hline O’Brien test & $14 / 2$ & $1 / 15$ & $p<0.05^{1}$ \\
\hline \multicolumn{4}{|c|}{ UCLA Shoulder Assessment Score (mean \pm SD) } \\
\hline Pain & $4.0 \pm 1.6$ & $8.2 \pm 1.5$ & $p<0.05^{2}$ \\
\hline Function & $4.8 \pm 2.0$ & $8.0 \pm 2.3$ & $p<0.05^{2}$ \\
\hline Active forward flexion & $4.8 \pm 0.4$ & $5.0 \pm 0.0$ & $p<0.05^{2}$ \\
\hline Strength of forward flexion & $4.3 \pm 0.4$ & $4.8 \pm 0.4$ & $p<0.05^{2}$ \\
\hline Patient satisfaction & $0.0 \pm 0.0$ & $5.0 \pm 0.0$ & $p<0.05^{2}$ \\
\hline Total score & $18.1 \pm 3.3$ & $31.3 \pm 3.7$ & $p<0.05^{2}$ \\
\hline UCLA score graded as Excellent & - & $35.0 \pm 1.1$ & - \\
\hline$(\%)$ & $(0 \%)$ & $(31.3 \%)$ & \\
\hline UCLA score graded as Good & - & $31.6 \pm 1.9$ & - \\
\hline$(\%)$ & $(0 \%)$ & $(43.8 \%)$ & \\
\hline UCLA score graded as Poor & $18.1 \pm 3.3$ & $26.3 \pm 2.1$ & - \\
\hline$(\%)$ & $(100 \%)$ & $(25.0 \%)$ & \\
\hline
\end{tabular}

${ }^{1}$ Results from McNemar test

${ }^{2}$ Results from Wilcoxon Signed Ranks test 